\title{
Placebo-Controlled Continuation Treatment with Mirtazapine: Acute Pattern of Response Predicts Relapse
}

\author{
Andrew A Nierenberg*,', Frederic M Quitkin ${ }^{2}$, Charlotte Kremer ${ }^{3}$, Martin B Keller ${ }^{4}$ and Michael E Thase ${ }^{5}$ \\ 'Havard Medical School, Massachusetts General Hospital, Boston, MA, USA; ${ }^{2}$ New York State Psychiatric Institute, Columbia University Medical \\ School, New York, NY, USA; ${ }^{3}$ Organon, Inc., USA; ${ }^{4}$ Department of Psychiatry, Brown University, USA; ${ }^{5}$ Western Psychiatric Institute and Clinic, \\ School of Medicine, University of Pittsburgh, USA
}

\begin{abstract}
Pattern of response to antidepressants has been proposed as a method to identify patients whose improvement is more likely due to drug vs those whose improvement on drug is more likely to be a placebo effect. It is hypothesized that those with 'true-drug initial response pattern' are most likely to benefit from continuation treatment. The relationship between acute patterns of response and subsequent placebo-controlled continuation treatment with the antidepressant mirtazapine is examined. A total of 410 outpatients were treated openly with mirtazapine for 8-12 weeks. Patients who remitted in the acute phase were randomized to continue the same dose of mirtazapine or switched to placebo. Acute phase responders were classified as 'placebo initial response pattern' (early responders and nonpersistent responders) and 'true-drug initial response pattern' (delayed and persistent responders). Of those with a 'true-drug initial response pattern,' I0/40 (25.0\%) relapsed with continuation mirtazapine, and 23/4I (56.1\%) relapsed when switched to placebo. The difference $(31.1 \%)$ is significant. Of those with a 'placebo initial response pattern,' 5/36 (13.9\%) relapsed with continuation mirtazapine, and I 2/39 (30.8\%) relapsed with placebo substitution. This difference (16.9\%) is not statistically significant. Moreover, the relapse rate for 'true-drug initial response pattern' patients switched to placebo (56.1\%) was also significantly greater than for 'placebo initial response pattern' patients switched to placebo (30.8\%). It has been suggested that patients with late onset and persistence are more likely to have improved because of drug. This hypothesis gains support from this study because of the different relapse rates of 'true-drug' responders on drug and placebo. The low relapse rate for patients with an acute placebo pattern switched to placebo suggests specific drug effect played a smaller role in their initial improvement.
\end{abstract}

Neuropsychopharmacology (2004) 29, I0I2-1018, advance online publication, 3 March 2004; doi: I0.1038/sj.npp. I 300405

Keywords: true-drug responders; placebo responders on drug; maintenance treatment; loss of drug effect; predictors of relapse; pattern analysis

\section{INTRODUCTION}

An enigma associated with virtually all antidepressants is inexplicable relapse during continuation or maintenance treatment (Byrne and Rothschild, 1998; Nierenberg and Alpert, 2000), a phenomenon referred to as tachyphylaxis. This is not an insignificant problem since recurrence rates vary from 15 to $40 \%$ in long-term clinical trials, a proportion mirrored in clinical practice (Coppen et al, 1978; Prien et al, 1973, 1984; Montgomery et al, 1988; Rouillon et al, 1989; Frank et al, 1990; Doogan and Caillard, 1992; Kupfer et al, 1992; Montgomery and Dunbar, 1993). Patients who relapse in spite of continuing their treatment may be demoralized and refuse further treatment.

*Correspondence: Andrew A Nierenberg, Massachusetts General Hospital, ACC 812, 15 Parkman Street, Boston, MA 02114, USA, Tel: + 617724 5600, Fax: + 6177267541 ,

E-mail: anierenberg@partners.org

Received 04 June 2003; revised 23 December 2003; accepted 06 January 2004

Online publication: 13 January 2004 at http://www.acnp.org/citations/ Npp0 I $130403250 /$ default.pdf
In addition to poor medication compliance, other possible causes of depressive breakthrough include tolerance and loss of acute placebo effect (Byrne and Rothschild, 1998; Nierenberg and Alpert, 2000). Pharmacodynamic and pharmacokinetic effects, changes in disease severity and depletion of a relevant effector substance have also been considered possible causes of tolerance (Mann, 1983; Lieb and Balter, 1984; Cohen and Baldessarini, 1985; Donaldson, 1989). Some evidence suggests that DOPA depletion may explain some drug benefit loss (McGrath et al, 1995). This paper examines the effect of an initial placebo-like pattern of response to antidepressants on subsequent relapse rates during maintenance therapy.

In clinical trials, typically $2 / 3$ of patients improve while taking antidepressants, and $1 / 3$ with placebo. Subtracting the placebo from the antidepressant response, one could reasonably conclude that of those who respond to antidepressants, about $1 / 3$ benefit from drug and $1 / 3$ from placebo-like effects. This suggests pharmacologic effects may explain half of improvement observed in drug-treated patients while nonspecific or placebo effects explain the other half. The Columbia Group (Quitkin et al, 1987) 
hypothesized that patients who improved after a 2-week delay and did not have a fluctuating course were more likely to have been receiving drug ('true-drug initial response pattern'). These patients are referred to as delayed persistent responders (referred to in this paper as 'truedrug initial response pattern'). Delayed persistent responses were three times more likely to occur on drug than placebo (Quitkin et al, 1987). Patterns, which included early onset or fluctuating improvement, occurred with equal frequencies on drug and placebo. It was also hypothesized that patterns characterized by improvement within the first 2 weeks of treatment or had fluctuating levels of improvement were more likely to be attributable to placebo effects. Specific rules to classify responses into specific drug $v s$ nonspecific patterns (placebo) have been published (Quitkin et al, 1987). In this paper, 'nonspecific placebo pattern' will be referred to as 'placebo initial response pattern' to simplify presentation.

Several groups have demonstrated that during acute treatment, delayed persistent responses were more likely to occur on drug than placebo (Fieve et al, 1986; Dunlop et al, 1990). Analysis of relapse rates during maintenance treatment offers the greatest support for the validity of pattern analysis. Stewart et al (1998) demonstrated that patients with 'true-drug initial response pattern' randomized to active drug relapsed less frequently than those switched to placebo. Even if judged to be in remission at study end, patients with 'placebo initial response pattern' had indistinguishable relapse rates after randomization to continue on either drug or placebo.

This body of data (Fieve et al, 1986; Quitkin et al, 1987; Dunlop et al, 1990; Stewart et al, 1998) suggest 'true-drug initial response pattern' identifies a group with a higher proportion whose improvement is due to the drug, and 'placebo initial response pattern' the converse, but these groups are not homogeneous. A group of patients with 'true-drug initial response pattern' probably contains some placebo responders, but a smaller proportion than a group of 'placebo initial response pattern.' Undoubtedly, some improvement in the 'placebo initial response pattern' is due to a drug effect but it is hypothesized placebo effects have increased relevance.

Since the relationship of the acute patterns of response and long-term treatment have only been studied with fluoxetine, we wished to determine if this is generalizable to other antidepressants. Furthermore, establishing validity of constructs such as 'true-drug pattern of response' requires multiple replications. A relapse prevention study with mirtazapine offered the opportunity to independently validate these observations.

In an attempt to assess placebo-like improvement on drug, four hypotheses were studied:

1. Patients who have a 'true-drug initial response pattern' will relapse more often while taking placebo than while continuing medication.

2. The relapse rates of patients with a 'placebo initial response pattern' will not differ for those randomized to mirtazapine or placebo.

3. For patients randomized to placebo, those with a 'true-drug initial response pattern' will have a higher relapse rate than those with a 'placebo initial response pattern.'

4. While continuing to receive mirtazapine, patients with a 'true-drug initial response pattern' will have a lower relapse rate than those with a 'placebo initial response pattern.'

\section{METHODS}

The study was performed as a multicenter trial, with patients enrolled at 12 US clinical research sites (see acknowledgments). Depressed patients were screened and included in the study if they had a diagnosis of major depressive episode according to DSM-IV criteria. In addition, patients (aged 18 years and older) had to be in reasonably good health and to have no history of drug or alcohol abuse for at least 3 months preceding their enrollment in the study. The details of the inclusion and exclusion criteria have been published (Thase et al, 2001).

Following consent, prospective patients began a 7-10-day single-blind placebo lead-in. The structured interview guide for the Hamilton Rating Scale for Depression (HRSD) was used (Williams, 1998). The first 17 items of the HRSD (Hamilton, 1960) was used to determine severity. Study entrance required a minimum score of 18 . An HRSD-17 score reduction of more than $20 \%$ during the $7-10$-day leadin period rendered a patient study ineligible.

Mirtazapine was administered during the acute phase at an initial dose of $15 \mathrm{mg}$ each day. Dose titration was permitted to $30 \mathrm{mg} /$ day after at least 1 week and to $45 \mathrm{mg} /$ day after at least 2 weeks of therapy. Patients were evaluated after $1,2,3,4,6$, and 8 weeks (with optional evaluations at 10 and 12 weeks) of therapy using the HRSD and the Clinical Global Impressions (CGI) scale (Guy, 1976). Remission was defined as HRSD-17 scores of $\leqslant 7$ and CGI scores of 1 or 2 (ie much or very much improved) sustained for at least 2 weeks. To meet these criteria, no fewer than 8 and no more than 12 weeks of treatment were permitted. Entry in the 40-week double-blind continuation phase protocol was limited to patients in remission.

Patients meeting remission criteria entered the doubleblind continuation phase were randomized to identically appearing placebo or mirtazapine tablets. As a result, approximately $50 \%$ of randomized patients were switched to placebo. Medication dosage was stable during the continuation phase - no further increases or decreases in dosage were allowed. Patients were evaluated at monthly intervals during the continuation phase, with interim visits permitted if clinically indicated.

The criterion for relapse was an investigators' decision that the patient was clinically depressed and required an immediate change in treatment.

\section{Statistical Analyses}

The study was designed to have an $80 \%$ chance to detect a difference in relapse rates of $25 \%$ or more $(p=0.05)$ between continuation mirtazapine and placebo groups. A remission rate of approximately $30 \%$ was anticipated during the acute phase. Therefore, 500 patients were enrolled in the 
acute phase in order to randomize 150 patients ( 75 per treatment group) in the continuation phase trial.

Published guidelines were used to classify 'true-drug initial response pattern' and 'placebo initial response pattern' observed during acute phase (Quitkin et al, 1987). Response patterns were based on the CGI Global Impression of Change Scale (Guy, 1976). 'True-drug initial response pattern' responders had a score of $<3$ (much or very much improved) for the first time in week 3 or later and then did not have a subsequent score of $\geqslant 3$ (minimally improvement to very much worse) during acute open treatment with mirtazapine. 'Placebo initial response pattern' responders were those subjects who had a CGI score $<3$ in weeks 1 or 2 or those who had a score $<3$ at any time point and then a score of $\geqslant 3$ in a subsequent visit. Outcome of 'true-drug initial response pattern' responders and 'placebo initial response pattern' responders during the double-blind placebo-substitution maintenance phase is the central focus of this paper.

The primary analyses for each hypothesis tested whether relapse during the 40 -week continuation study differed between the comparison groups. The log-rank test assessed Kaplan-Meier survival curves for differences in time to relapse between groups. Since there are two group hypotheses, we did not test for interactions and present the survival curves in two-group contrasts.

Analyses testing hypothesis one compared relapse for patients with 'true-drug initial response pattern' randomized to placebo $(N=41)$ with those randomized to drug $(N=40)$.

For hypothesis two, analyses compared relapse for patients with 'placebo initial response pattern' randomized to placebo $(N=39)$ with those randomized to drug $(N=36)$

Analyses testing hypothesis three compared relapse for patients randomized to placebo with 'true-drug initial response pattern' $(N=41)$ vs those with a 'placebo initial response pattern' $(N=39)$.

Analyses testing hypothesis four compared patients randomized to drug with a 'true-drug initial response pattern' $(N=40)$ vs those with a 'placebo initial response pattern' $(N=36)$.

\section{RESULTS}

A total of 156 remitted patients agreed to enter the doubleblind discontinuation phase. The overall data from this study have been presented (Thase et al, 2001).

Among those randomized in the double-blind portion of the study, $75(49.1 \%)$ were classified as placebo responders and $81(50.9 \%)$ as 'true-drug initial response pattern'. In all, 76 were randomized to continue to take mirtazapine and 80 randomized to switch to placebo. Demographic and baseline variables were previously published (Thase et al, 2001). In summary, the 75 patients with acute placebo pattern were $47 \%$ female, mean age $39.7 \pm 11.7$ years with an age of onset of $24.2 \pm 12.4$ years; all had major depressive disorder and were in remission at the start of the continuation study. The 81 patients with 'acute true-drug pattern' were $54 \%$ female with a mean age of $41.1 \pm 11.5$ years and an age of onset of $20.7 \pm 11.4$ years; all had major depressive disorder and were in remission at the start of the continuation study.
There were no statistically significant differences in demographic characteristics.

Although the primary analyses are the log-rank tests assessing Kaplan-Meier survival curves, we first present a bar graph so that an overview of the data are available (Figure 1). Patients with a 'true-drug initial response pattern' switched to placebo had a 56\% (23/41) relapse rate; those continued on drug had a $25 \%(10 / 40)$ relapse rate $\left(\chi^{2}=6.8, \mathrm{df}=1, p=0.009\right)$. Patients with a 'placebo initial response pattern' switched to placebo had a 30\% (12/39) relapse rate; those continued on drug had a $14 \%(5 / 36)$ relapse rate. This was not significant $\left(\chi^{2}=2.1, \mathrm{df}=1\right.$, $p=0.14$ ). Differences in relapse rates for those patients assigned to placebo for the 'true-drug initial response pattern' $v s$ the 'placebo initial response pattern' are significant $\left(\chi^{2}=4.2, \mathrm{df}=1, p=0.03\right)$. Differences in relapse rates for the two groups assigned to drug are not significant $\left(\chi^{2}=2.15, \mathrm{df}=1, p=0.14\right)$.

\section{Test of Hypotheses}

Do patients with a 'true-drug initial response pattern' relapse more often while receiving placebo substitution than while receiving continued mirtazapine therapy? Among patients with a 'true-drug initial response pattern,' survival was greater for patients receiving mirtazapine than placebo (see Figure 2). Kaplan-Meier survival analyses for weeks $0-40$ demonstrated a significantly higher relapse rate in placebo-treated patients, log-rank test score $8.55, \mathrm{df}=1$, $p=0.003$.

Do patients with a 'placebo initial response pattern' relapse more often while receiving placebo than continued mirtazapine therapy?. The Kaplan-Meier analyses did not demonstrate significantly greater relapse with the administration of placebo than with the administration of continued mirtazapine therapy (see Figure 3), log-rank test $=3.23$,

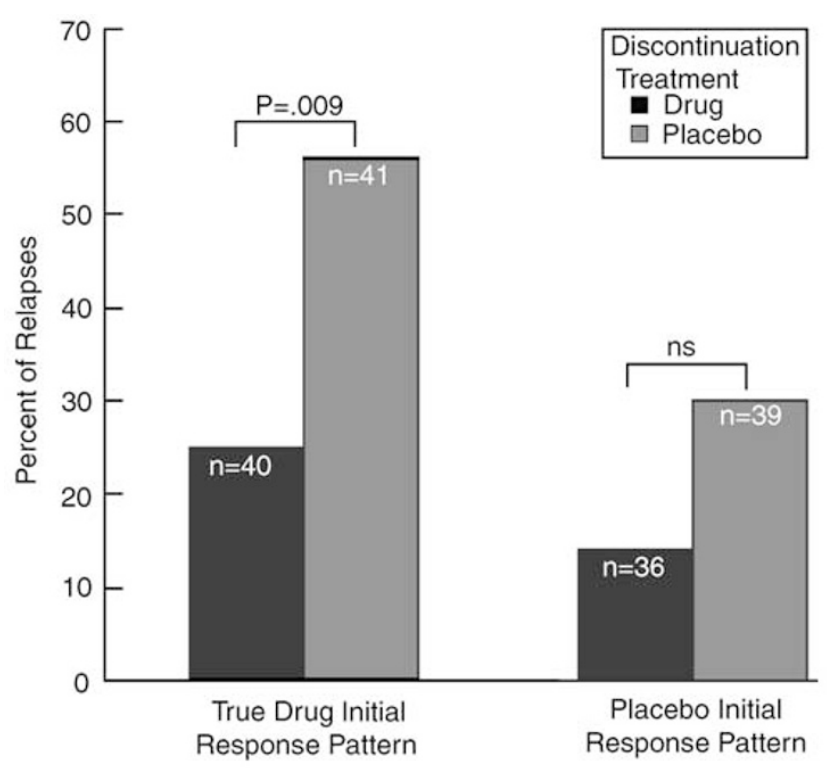

Acute Phase Response Pattern

Figure I Acute phase response pattern. 


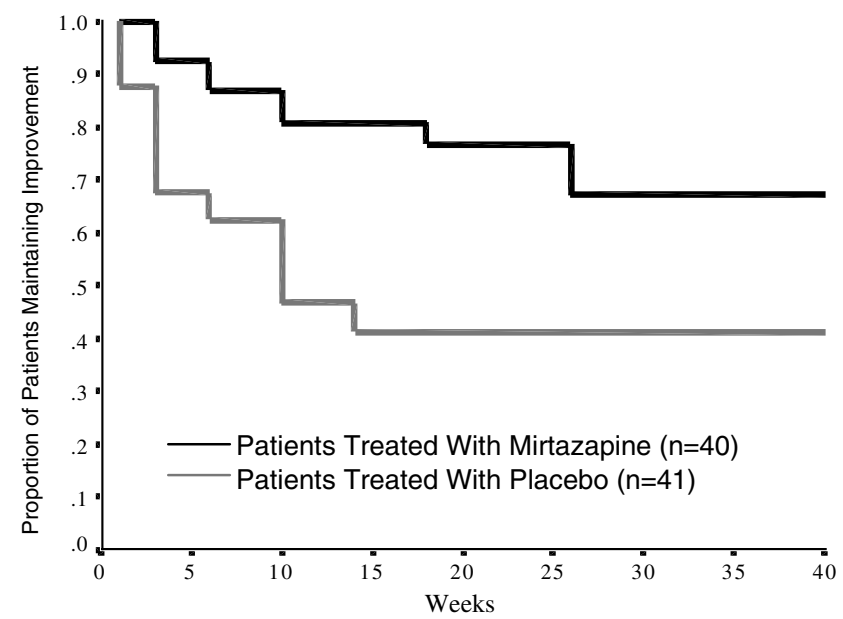

Figure 2 Kaplan-Meier survival curves comparing survival from weeks 0 to 40 among patients with a 'true-drug' short-term response pattern; the patients were treated with mirtazapine vs placebo (log-rank test score, 8.55, $\mathrm{df}=\mathrm{I}, \mathrm{p}=0.0034)$

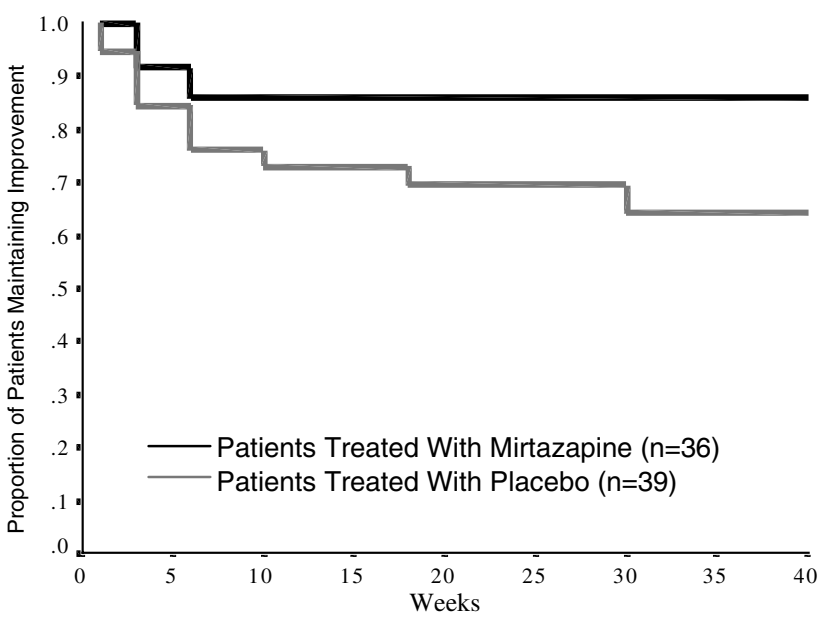

Figure 3 Kaplan-Meier survival curves comparing survival from weeks 0 to 40 among patients with a 'placebo' short-term response pattern; the patients were treated with mirtazapine vs placebo (log-rank test score, 3.23, $\mathrm{df}=\mathrm{I}, \mathrm{p}=0.0722$ ).

$\mathrm{df}=1, p=0.072$. With an $N$ of 40 and an effect size of 0.4 (which is what we observed), there is approximately a $60 \%$ chance of demonstrating a statistical difference between groups. This is an underpowered analysis; significant differences may have been found with a larger group of 'nonspecific' responders randomized to drug or placebo. This issue is discussed further below.

Do patients randomized to placebo with a 'true-drug initial response pattern' have a higher relapse rate than those with a 'placebo initial response pattern?'. In weeks $0-40$, patients with a 'true-drug initial response pattern' demonstrated significantly more relapse if assigned to placebo than patients with a 'placebo initial response pattern' randomized to placebo (see Figure 4), log-rank test score $=4.87, \mathrm{df}=1, p=0.027$ ).

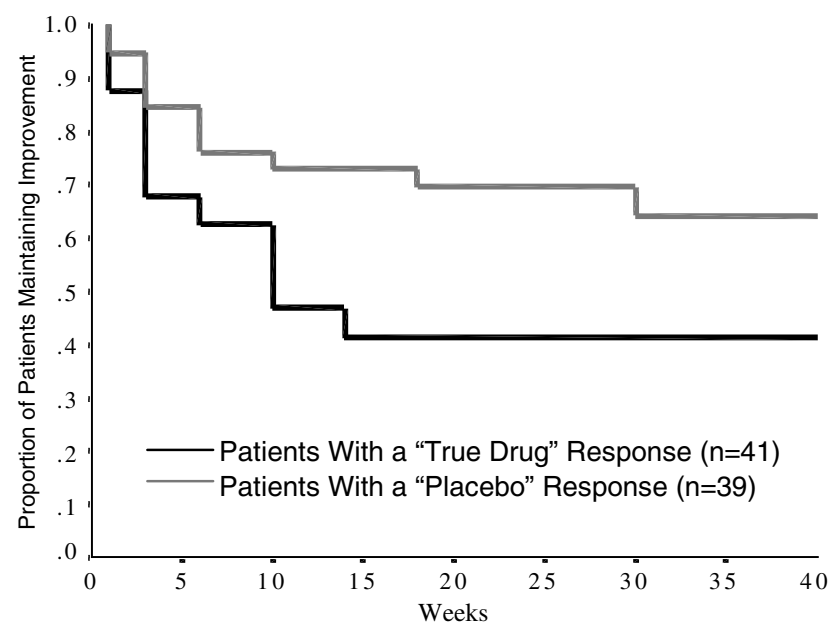

Figure 4 Kaplan-Meier survival curves of patients treated with placebo from weeks 0 to 40 comparing survival of those with a 'true-drug' vs a 'placebo' short-term response pattern (log-rank test score, 4.87, df =I, $p=0.0273)$.

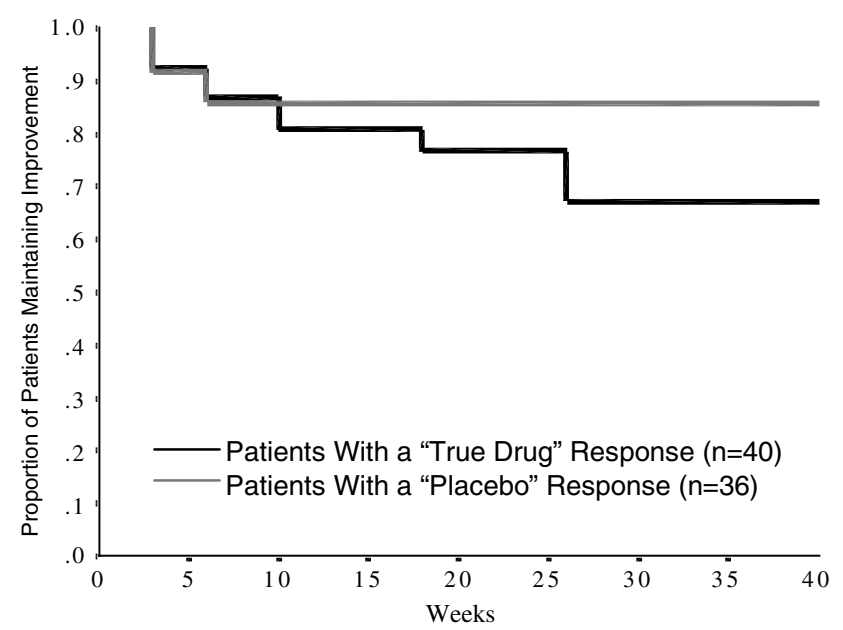

Figure 5 Kaplan-Meier survival curves of patients treated with mirtazapine from weeks 0 to 40 comparing survival of those with a 'truedrug' vs a 'placebo' short-term response pattern (log-rank test score, I.56, $\mathrm{df}=\mathrm{I}, p=0.2110)$

Do patients randomized to drug with a 'placebo initial response pattern' have a higher relapse rate than patients with a 'true-drug initial response pattern?'. In weeks 0-40, for patients randomized to mirtazapine, those with a 'truedrug initial response pattern' had a relapse rate that did not differ from patients on drug with a 'placebo initial response pattern,' log-rank test score $=1.56, \mathrm{df}=1, p=0.211$ (see Figure 5).

\section{DISCUSSION}

These data suggest that response patterns are inexact, and 'true-drug initial response patterns' and 'placebo initial response patterns' are heterogeneous. Among patients with a 'true-drug initial response pattern,' some improvement may be due to placebo effects. Alternatively, among patients 
with 'placebo initial response patterns,' some improvement is probably attributable to medication. The present study appears to replicate the observation of Stewart et al that pattern analysis identifies patients who are most likely to benefit from continuing medication, that is, those with a 'true-drug initial response pattern'. A corollary of this is that their initial improvement is more likely to be due to a drug effect.

Stewart et al (1998) found that patients who had a 'true-drug initial response pattern' and received maintenance fluoxetine had the best prognosis. In comparison, three groups had inferior and roughly equivalent outcome: patients with (1) 'true-drug initial response pattern' switched to placebo, (2) 'placebo initial response pattern' maintained on drug, or (3) 'placebo initial response pattern' switched to placebo. Continuation on drug was only beneficial for 'true-drug initial response pattern'.

Therefore, based on these earlier results, we anticipated that patients in the present study with 'true-drug initial response pattern' would have lower relapse rates if assigned to drug rather than placebo; this was observed. It was also anticipated that 'placebo initial response pattern' patients would have no statistically significant differences in relapse rates during the continuation phase whether assigned to drug or placebo. This is true, but it should be noted that this comparison is underpowered and that the relapse rates for the 'placebo-pattern' group was about $31 \%$ for those switched to placebo and only about $14 \%$ for those continued on mirtazapine, a difference of about $17 \%$. This difference may have been statistically significant with a larger $N$. It is possible that some patients with 'placebo initial response patterns' may have benefited from continuing mirtazapine; although the $17 \%$ difference in relapse rates between the 'placebo initial response patterns' placebo-switch and continued mirtazapine groups was substantially less than the $31 \%$ difference in relapse rates in those with a 'true-drug initial response pattern' randomized to switch to placebo or continue on mirtazapine. In other words, the effect size for relapse rates during continued mirtazapine compared to a switch to placebo was almost twice as large for the 'truedrug initial response pattern' group compared to the 'placebo initial response pattern' group.

We also observed that for all patients assigned to placebo, those who initially responded with a 'true-drug initial response pattern,' had significantly greater relapse rate (56\%) than those with a 'placebo initial response pattern' (30\%). This finding also supports the hypothesis that patients with a 'true-drug initial response pattern' are more likely to have improved because of specific drug effect. This follows because it suggests more patients with a 'true-drug initial response pattern' improved because of a pharmacologic effect. When the drug was removed, there is greater relapse rate in the group with 'true-drug initial response pattern' than the group with a 'placebo initial response pattern.' One could reasonably conclude that improvement in the 'placebo initial response pattern' group was less likely to stem from a pharmacologic effect.

Patients who were randomized to continue taking mirtazapine did well regardless of their initial response pattern. How do we explain the low relapse rate in the patients with a 'placebo initial response pattern' randomized to drug in the mirtazapine study? In the Stewart et al study, the relapse rate for patients with a 'placebo initial response pattern,' regardless of maintenance drug, was high, approximately $50 \%$ relapsing during a 14 -week observation. Prior studies suggest that chronic or more severely ill patients randomized to placebo are more likely to relapse than nonchronic or less severely ill patients (Harrison et al, 1986; Stewart et al, 1989, 1997). A plausible explanation for the differences in relapse rate with drug for those with 'placebo initial response pattern' in the two studies is that patients in the mirtazapine study (compared to Stewart et al) had a lower proportion of chronically or severely ill patients. This assertion is supported by the low relapse rate for patients with 'placebo initial response pattern' continued on drug (approximately 14\%). This may also explain the relatively low relapse rate (approximately $30 \%$ ) for patients in the mirtazapine study with a 'placebo initial response pattern' switched to placebo. This suggests that outcome during continuation treatment depends on an interaction between illness characteristics, type of treatment, and 'initial response pattern.' Patients with 'placebo initial response pattern' may have low relapse rates if their illness is not chronic or is characterized by infrequent exacerbations. The fact that the relapse rate was lower for all patients with placebo initial response pattern $(23 \%(17 / 75))$ in comparison to true-drug pattern $(41 \%(33 / 81)), \chi^{2}=5.04$, $\mathrm{df}=1, p<0.05$, is consistent with the view that patients with different biology exhibit initial drug and initial placebo responses.

The relevance of a 'true-drug initial response pattern' is that it predicts drug-placebo differences during maintenance. Patients with a 'placebo initial response pattern' may have a high or low relapse rate, but there should be a little difference with drug and placebo. This was observed in the Stewart et al study and the present study. The fact that only two studies support these assertions makes this tentative, requiring replication. Note, however, that data regarding chronicity or severity are not available to actually compare the two studies and reasons for the differences between the studies should be considered speculative.

Do patients on maintenance antidepressants relapse because of loss of placebo effect? The Stewart et al paper directly supports this hypothesis because relapse for patients with 'placebo initial response pattern' maintained on fluoxetine was approximately $20 \%$ greater than for patients with 'true-drug initial response pattern.' The present study indirectly supports this hypothesis-since there was no statistically significant difference in relapse rates for patients with 'placebo initial response pattern' maintained on drug and placebo, some of the improvement observed in this group must be due to placebo effects. The fact that relapse on placebo was statistically higher for patients with 'truedrug initial response pattern' vs 'placebo initial response pattern' also suggests that patients' improvement is based on a different mechanism. The mirtazapine study supports the hypothesis that (1) some patients' improvement does not depend on a drug effect but on a placebo effect, and (2) some relapse on drug will be due to loss of placebo effect. The mirtazapine study does not permit a quantitative estimate because patients with a 'placebo initial response pattern' did well on drug and placebo.

Pattern analysis should be considered in planning continuation studies. In the present study, if all patients 
who continue taking either drug or placebo are considered, the difference in relapse rates is $25 \%$ (relapse on drug $19 \%$ $(15 / 76)$, relapse on placebo $44 \%(35 / 80))$. If the analysis is limited to patients with 'true-drug initial response pattern', the difference is $31 \%$ (relapse on drug $25 \%$ $(10 / 40)$, relapse on placebo $56 \%(23 / 41))$. In contrast, if the analysis is limited to patients with 'placebo initial response pattern', the difference in relapse rates for those continued on drug compared to placebo is only $17 \%$ (relapse on drug 14\% (5/36), relapse on placebo $31 \%$ (12/ 39)). These results suggest that stratification by initial response patterns prior to randomization in the continuation phase may be informative for future studies of relapse prevention.

A limitation of this study is that the main goal of the clinical trial was to assess the long-term efficacy of mirtazapine using a placebo-substitution paradigm (Thase et al, 2001). The analysis of patterns of response and subsequent course after placebo substitution or continued mirtazapine was post hoc. A more robust design would have been a placebo-controlled acute trial followed by a controlled maintenance phase. Nonetheless, the open acute trial of mirtazapine does have ecological validity and approaches clinical practice.

Heuristically, pattern analysis appears to identify groups with a different prognosis. One group appears to have a greater proportion whose improvement is due to a 'truedrug effect.' This should be considered in studying the mechanisms for how drugs work. Including patients with nonspecific improvement in such studies may obfuscate identification of relevant mechanisms. Clinicians may wish to consider patterns of response to acute treatment when evaluating patients who have reason not to continue drug, such as intolerable side effects or desire to become pregnant. The present study and the Stewart et al study suggest that patients with a 'placebo initial response pattern' might have minimal protection for depressive relapse with continued drug compared to switching to placebo. Pattern analysis is only one parameter and should be considered in the context of all relevant clinical variables such as prior suicide attempts, number of previous episodes, and family history. The Stewart et al study and the current study suggest patients with 'true-drug initial response pattern' are at greatest risk of relapse if drug is prematurely withdrawn and may benefit the most from continuation pharmacotherapy.

\section{ACKNOWLEDGEMENTS}

This study was supported in part by a series of grants from Organon Inc., West Orange, NJ. The authors thank the following investigators for their contribution to this study: James D Bremner, MD, Bremner Research Institute, Olympia, WA; Alan D Feiger, MD, Feiger PsychMed Center, Wheat Ridge, CO; Ronald $\mathrm{R}$ Fieve, MD, Fieve Clinical Services, New York, NY; James M Ferguson, MD, Pharmacology Research Corporation, Salt Lake City, UT; Edward S Friedman, MD, and Robert H Howland, MD, Department of Psychiatry, University of Pittsburgh School of Medicine, Pittsburgh, PA; Barbara L Kennedy, MD, PhD, Department of Psychiatry and Behavioral Sciences, School of Medicine,
University of Louisville, Louisville, KY; Gabor Keitner, MD and Van Miller, PhD, Brown University Medical School, Providence, Rhode Island; Fred W Reimherr, MD, Department of Psychiatry, University of Utah School of Medicine, Salt Lake City, UT; Ward T Smith, MD, Pacific Northwest Clinical Research Center, Portland, OR; Stephen M Stahl, MD, Institute for Psychopharmacology Research, San Diego, CA; Kenneth J Weiss, MD, Delaware Valley Research Associates, Inc., Conshohocken, PA.

\section{REFERENCES}

Byrne SE, Rothschild AJ (1998). Loss of antidepressant efficacy during maintenance therapy: possible mechanisms and treatments. J Clin Psychiatry 59: 279-288.

Cohen B, Baldessarini R (1985). Tolerance to therapeutic effects of antidepressants. Am J of Psychiatry 142: 489-490.

Coppen A, Ghose K, Montgomery S et al (1978). Continuation therapy with amytriptiline in depression. Br J Psychiatry 133: 28-33.

Donaldson S (1989). Tolerance to phenelzine and subsequent refractory depression: three cases. J Clin Psychiatry 50: 33-35.

Doogan D, Caillard V (1992). Sertraline in the prevention of depression. Br J Psychiatry 160: 217-222.

Dunlop S, Dornseif B, Wernicke F, Potvin J (1990). Pattern analysis shows beneficial effect of fluoxetine treatment in mild depression. Psychopharmcol Bull 26: 173-180.

Fieve R, Goodnick P, Peselow E, Barouche F, Schlegel A (1986). Pattern analysis of antidepressant response to fluoxetine. J Clin Psychiatry 47: 560-562.

Frank E, Kupfer D, Perel J, Cornes C, Jarrett DB, Mallinger AG et al (1990). Three-year outcomes for maintenance therapies in recurrent depression. Arch Gen Psychiatry 47: 1093-1099.

Guy W (1976). ECDEU Assessment Manual for Psychopharmacology, Revised, US Department of Health, Education, and Welfare (ADM) 76-38. In: Rockville MD (ed). National Institute of Mental Health: Rockville, MD. pp 218-222.

Hamilton M (1960). A rating scale for depression. J Neurol Neurosurg Psychiatry 23: 56-62.

Harrison W, Rabkin J, Stewart JW, McGrath PJ, Tricamo E, Quitkin FM (1986). Phenelzine for chronic depression: a study of continuation treatment. J Clin Psychiatry 47: 346.

Kupfer D, Frank E, Perel JM, Cornes C, Mallinger AG, Thase ME et al (1992). Five-year outcome for maintenance therapies in recurrent depression. Arch Gen Psychiatry 49: 769-773.

Lieb J, Balter A (1984). Antidepressant tachyphylaxis. Med Hypotheses 15: 279-291.

Mann J (1983). Loss of antidepressant effect with long-term monoamine oxidase inhibitor treatment without loss of monoamine oxidase inhibition. J Clin Psychopharmacol 3: 363-366.

McGrath P, Quitkin FM, Klein DF (1995). Bromocriptine treatment of relapses seen during selective serotonin re-uptake inhibitor treatment of depression. J Clin Psychopharmacol 4: 289-291.

Montgomery SA, Dufour H, Brion S, Gailledreau J, Laqueille X, Ferrey $\mathrm{G}$ et al (1988). The prophylactic efficacy of fluoxetine in unipolar depression. Br J Psychiatry 153(Suppl 3): 69-76.

Montgomery S, Dunbar G (1993). Paroxetine is better than placebo in relapse prevention and the prophylaxis of recurrent depression. Int Clin Psychopharmacol 8: 189-195.

Nierenberg AA, Alpert JE (2000). Depressive breakthrough. Psychiatric Clin North Am 23: 731-742.

Prien RF, Kupfer DJ, Mansky PA, Small JG, Tuason VB, Voss CB et al (1984). Drug therapy in the prevention of recurrences in unipolar and bipolar affective episodes. Arch Gen Psychiatry 41: 1096-1104. 
Prien R, Mett J, Caffey E (1973). Lithium carbonate and imipramine in prevention of affective episodes. Arch Gen Psychiatry 29: 420-425.

Quitkin FM, Rabkin JD, Markowitz JM, Stewart JW, McGrath PJ, Harrison W (1987). Use of pattern analysis to identify true-drug response. Arch Gen Psychiatry 44: 259-264.

Rouillon F, Phillips R, Serruier D, Ansart E, Gerard MJ (1989). Recurrence of unipolar depression and efficacy of maprotiline. Encephale 15: 527-534 (in French).

Stewart J, Greenfield S, Hays RD, Wells K et al (1989). Functional status and well-being of patients with chronic conditions: results from the Medical Outcome Study. JAMA 262: 907-913.

Stewart JW, Quitkin FM, McGrath PJ, Amsterdam J, Fava M, Fawcett J et al (1998). Use of pattern analysis to predict differential relapse of remitted patients with major depression during 1 year of treatment with fluoxetine or placebo. Arch Gen Psychiatry 55: 334-343.

Stewart JW, Tricamo E, McGrath PJ, Quitkin FM (1997). Prophylactic efficacy of phenelzine and imipramine in chronic atypical depression: likelihood of recurrence on discontinuation after 6 months' remission. Am J Psychiatry 154: 31-36.

Thase ME, Nierenberg AA, Keller MB, Panagides J, The Relapse Prevention Study Group (2001). Efficacy of mirtazapine for prevention of depressive relapse: a placebo-controlled doubleblind trial of recently remitted high-risk patients. J Clin Psychiatry 62: 782-788.

Williams JBW (1998). A structured interview guide for the Hamilton Depression Rating Scale. Arch Gen Psychiatry 45: 742-747. 